\title{
Impact of 12 Weeks Practice of Chandranadi Pranayama on Cardiac Autonomic Functions, Perceived Stress Score and Biochemical Parameters in Pre-hypertensive Young Adult Males- A Pilot Study
}

\author{
Aswathy Viswanathan ${ }^{1}$, Velkumary Subramanian ${ }^{1, *}$, Gopal Krushna Pal' ${ }^{2}$, Keni Gowsi ${ }^{1}$
}

\section{Aswathy Viswanathan', Velkumary Subramanian ${ }^{1, *}$, Gopal Krushna Pal ${ }^{2}$, Keni Gowsi ${ }^{1}$ \begin{abstract}
Institute of Post Graduate Medical Education and Research, Puducherry, INDIA.

2Program Director, Advance Center for Yoga, Senior Professor, Department of Physiology, Jawaharlal Institute of Post Graduate Medical Education and Research, Puducherry, INDIA.
\end{abstract} \\ 'Department of Physiology, Jawaharlal}

\section{*Correspondence \\ Dr. Velkumary Subramanian Additional Professor, Department of Physiology, JIPMER, Puducherry-605 006, INDIA. \\ Phone: +91- 9442213592 \\ Email: velkumary@gmail.com \\ History \\ - Submission Date: 23-07-2020; \\ - Review completed: 20-08-2020; \\ - Accepted Date: 12-09-2020}

DOI : 10.5530/ijcep.2020.7.3.27

Article Available online

http://www.ijcep.org

\section{Copyright}

(C) 2020 Phcog.Net. This is an openaccess article distributed under the terms of the Creative Commons Attribution 4.0 International license.

\begin{abstract}
Background and Aim: Prehypertensive subjects pose significant future cardiovascular (CV) risk and lifestyle modification is considered as the first line of intervention for them. Few scientific trials revealed that regular composite yoga practices restore hemodynamic stability and reduces the CV risk in various diseases. However, there is a dearth of data on single yoga practice on cardiovascular health. Hence this study was undertaken to assess the impact of single slow pranayama (Chandranadi pranayama) practice for 12 weeks on cardiac autonomic functions, perceived stress score (PSS) and serum nitric oxide. Methods: The outcome parameters were recorded in thirty male prehypertensive subjects in the age group of 18 to 40 years at baseline and after intervention. They were divided into two groups, designated as control and pranayama group with fifteen subjects in each group. Pranayama group was given 27 rounds of chandranadi pranayama practice, an exclusive left nostril breathing followed by relaxation in shavasana for 10 min. The intervention was given for a period of twelve weeks. The parameters recorded were HR, BP, short-term heart rate variability (HRV), baroreflex sensitivity (BRS), PSS and the cardiac autonomic reactivity tests which included heart rate response to standing (30:15 ratio), heart rate response to deep breathing ( $\mathrm{E}: \mathrm{I}$ ratio) and isometric handgrip test ( $\triangle \mathrm{DBDisg})$. Further, $3 \mathrm{ml}$ of venous blood was collected for serum nitric oxide and uric acid assay. Results: A significant decrease in PSS, $H R, S B P, D B P$ and LF/HF ratio of frequency domain indices of HRV and a significant increase in BRS, 30:15 ratio, E:I ratio and serum nitric oxide was noted in pranayama group at 12 weeks, when compared with their baseline data. No significant changes were observed in control group at 0 and 12 weeks. Conclusion: In our study, 12 weeks of exclusive left nostril breathing practice found to normalize BP and HR, improved BRS, restored sympathovagal balance and reduced perceived stress in prehypertensive subjects, which signifies decreased $\mathrm{CV}$ risk in them.

Key words: Prehypertension, Perceived stress score, HRV, BRS, Autonomic reactivity test.
\end{abstract}

\section{INTRODUCTION}

Hypertension is one of the leading noncommunicable diseases in India, with an increased risk of developing cardiovascular and cerebrovascular morbidities on long standing uncontrolled state. [1] Pre-hypertension is the precursor of clinical hypertension. As per the Seventh Joint National Committee (JNC-7) classification, pre-hypertension is defined as, systolic blood pressure (SBP) of 120 to $139 \mathrm{mmHg}$ and/ or diastolic blood pressure (DBP) of 80 to $89 \mathrm{~mm} \mathrm{Hg}$, based on two or more correctly measured, seated BP readings on each of two or more office visits. ${ }^{[2]}$ Pre-hypertensives are reported to be at high risk of developing overt hypertension and adverse cardiovascular events compared to normotensive subjects. ${ }^{[3]}$ Further, pre-hypertension is relatively more common in

young adults, especially in those who have a family history of hypertension. ${ }^{[4]}$ The overall prevalence of pre-hypertension in adult is $31 \%$ and found to be more common in males. ${ }^{[5]}$ According to Gupta et al. the prevalence of prehypertension in adult males is $40.2 \%$ and in females, it is $30.1 \% .^{[6]}$

Although the etiology of hypertension is multifactorial, psychosocial stress is frequently implicated in the development of hypertension in youngsters. ${ }^{[7-9]}$ Any mental stress can cause an abnormal activation of the sympathetic nervous system resulting in autonomic imbalance leading to dysregulation of BP. ${ }^{[10,11]}$ Further research studies had documented the association of sympathovagal imbalance in the form of sympathetic overactivity and vagal withdrawal, contributing to 
the development of pre-hypertension and hypertension. ${ }^{[12-14]}$ The Framingham heart study has indicated that $50 \%$ of pre-hypertensives, below the age of 65 developed hypertension within a span of 4 years, if not addressed. ${ }^{[4]}$ Hence, pre-hypertensive are at high risk for overt hypertension compared to normotensive subjects. Lifestyle modification is considered as the first line of therapy in prehypertension, which include stress reduction strategies, improving physical activity, weight management and dietary changes. ${ }^{[15]}$

Yoga is a mind body therapy originated from India, which brings holistic health to the practitioner. Scientific data discloses that regular yoga training, especially the yogic breathing (pranayama) is beneficial in reducing the stress and restoring the sympathovagal homeostasis. [16] Pranayama is the fourth limb of Asthanga yoga, which is a breath manipulation technique that improves the cardiovascular health, on regular practice. ${ }^{[17]}$ Particularly, the slow pranayamic breathing practices are proclaimed to be more beneficial in this aspect as reported by Pal, et $a l .{ }^{[18]}$ Studies have also demonstrated that the specific nostril breathing can alter autonomic functions in healthy adults. ${ }^{[19]}$ Raghuraj, et al. affirmed, increased sympathetic activity and BP following right nostril breathing and decreased sympathetic activity and blood pressure on practice of left nostril breathing. ${ }^{[20]}$ Further, Bhavanani, et al. have observed the acute effect of chandranadi pranayama (CNP) practice, an exclusive left nostril breathing, in reducing blood pressure in hypertensive patients, by modulating the autonomic activity of cardiovascular system. ${ }^{[21]}$ Moreover, impaired nitric oxide (NO) is implicated in the development and maintenance of hypertension. ${ }^{[22]}$ Gamboa, et al. revealed that NO suppress sympathetic vascular tone. Whereas endothelial dysfunction due to varied etiology with reduced NO contribute to sympathetic nerve activation (SNA) and hence development of hypertension, ${ }^{[23]}$ conversely SNA also impair nitric oxide function. ${ }^{[24]}$ Though previous studies have focused on the integrated yoga therapy in controlling hypertension, there is paucity of scientific data on the impact of single slow pranayamic breathing on cardiovascular health, stress level in prehypertensive subjects. Hence, this study was undertaken to assess the effect of 12 weeks of exclusive chandranadi pranayama practice on the cardiac autonomic functions, perceived stress score and blood parameters in young male pre-hypertensive adults.

\section{MATERIALS AND METHODS}

\section{Setting}

This randomized control study was conducted in prehypertensive adults to assess the impact of 12 weeks CNP practice on cardiac autonomic functions, perceived stress score and serum nitric oxide, uric acid. The study protocol was approved by the Institutional Ethics Committee for human studies (Approval No: JIP/IEC/2016/1149). The study was conducted in the department of Physiology, JIPMER, Puducherry.

\section{Participants}

Thirty male pre-hypertensive patients in the age group of 18 to 40 years were recruited for the study, after following inclusion and exclusion criteria. These patients were randomized into control group and pranayama group. The pre-hypertensive subjects of the pranayama group were given 27 rounds of chandranadi pranayama practice twice a day for 12 weeks and the control group was not given any intervention.

\section{Inclusion Criteria}

Male subjects with SBP 120-139 mmHg and/or DBP 80-89 mmHg, in the age groups of 18-40 years, with BMI between 18.5 and $29.9 \mathrm{~kg} / \mathrm{m}^{2}$ and willing to practice pranayama were included in the study.

\section{Exclusion Criteria}

Pre-hypertensive with history of smoking and those who were on treatment for diabetes, cardiovascular disease, endocrine disorder, renal disease and on drugs that influence ANS activity were excluded. Those subjects who were receiving treatment for prehypertension and those who were already practicing yoga or other biofeedback therapy were also excluded from the study. The subjects were recruited by conducting a screening camp. They were screened for blood pressure using a digital BP monitor (Citizen CH 432B). Participants who satisfied the inclusion and exclusion criteria and were willing to take part in the study were recruited.

Number of groups studied: Two groups

Pranayama group $(n=15)$ : pre-hypertensive subjects willing for pranayama practice

Control group $(n=15)$ : pre-hypertensive subjects not on pranayama practice

After approval from the institute ethics committee, a written informed consent was obtained from all the study participants. They were instructed to report to the Autonomic Function Test lab in the department of Physiology, two hours after having a light breakfast and were asked to refrain from caffeine/ alcoholic beverages, $12 \mathrm{hr}$ before the recording. The following assessment was done between 9 a.m. and 11 a.m. The laboratory temperature was maintained at $\pm 24^{\circ} \mathrm{C}$.

\section{Anthropometric Parameters}

The baseline anthropometric parameters like height, weight and body mass index (BMI) and waist-hip ratio (WHR) were measured. A wallmounted stadiometer (VM Electronics Hardware Ltd) was used to measure the height accurate to the nearest $0.1 \mathrm{~cm}$. Weight was measured using a digital weighing machine (Charder Electronic Co. Ltd. Taichung, Taiwan, 2013) accurate to the nearest $0.1 \mathrm{~kg}$. BMI was calculated using the Quetelet index - weight $(\mathrm{kg}) /$ height $\left(\mathrm{m}^{2}\right)$ and WHR was calculated using the formula WHR = waist circumference $\mathrm{cm} /$ hip circumference $\mathrm{cm}$.

\section{Baseline Cardiovascular Parameters}

Basal blood pressure (BP) and heart rate (HR) was measured after 10 min of rest in sitting posture. BP was recorded by oscillometric method using automated digital BP monitor Omron MX3 (Omron Healthcare Co. Ltd, Kyoto, Japan). Recordings were taken thrice with 2-min of rest in between and the average was taken as the final BP reading. Pulse Pressure (PP), Mean Arterial Pressure (MAP), Rate Pressure Product (RPP) was calculated using the formula, $\mathrm{PP}=\mathrm{SBP}-\mathrm{DBP}, \mathrm{MAP}=\mathrm{DBP}+1 / 3 \mathrm{PP}, \mathrm{RPP}=$ (BHR X SBP) $\mathrm{X}^{-2}$ respectively.

\section{Recording of Short Term Heart Rate Variability (HRV)}

Short-term HRV analysis is a convenient method for the estimation of autonomic tone, it was recorded in the study, as per the standard guidelines recommended by Task Force for short-term HRV measurement. ${ }^{[25]}$ After $10 \mathrm{~min}$ of supine rest, lead II ECG was recorded for $5 \mathrm{~min}$ and sample acquisition was done at a rate of $1000 \mathrm{~Hz}$ /sec using BIOPAC MP 150 data acquisition system (BIOPAC Inc., Goleta, CA, USA). The data was transferred from BIOPAC to a windows-based PC with Acq Knowledge software version 3.8.2. Ectopic and artefacts were removed manually from the recorded ECG. RR tachogram of 5 min ECG was extracted and HRV analysis was done using the Kubios HRV analysis software version 1.1 (Bio-signal Analysis Group, Kuopio, Finland). Frequency domain indices (FDI) were computed using Fast Fourier Transformation, which classify HRV spectrum into three frequency bands, very low frequency $(\mathrm{VLF}<0.04)$, low frequency $(\mathrm{LF}-0.04-0.15 \mathrm{~Hz})$ and high frequency 
(HF - 0.15-0.4 Hz) $(13,14)$ bands, both in absolute powers given as $\mathrm{ms}^{2}$ and in normalized unit. Normalized HF power was calculated using the formula HF nu=HF/(TP-VLF)X100, which denotes parasympathetic activity and normalized LF power $(\mathrm{LF} \mathrm{nu})=\mathrm{LF} /(\mathrm{TP}-$ VLF) X 100 denotes predominantly sympathetic, also include vagal and baroreflex activity. LF/HF ratio indicates sympathovagal balance and Total power $(\mathrm{TP}=\mathrm{VLF}+\mathrm{LF}+\mathrm{HF})$ represent the overall variability. The time domain indices (TDI) were computed by statistical method which include, mean and standard deviation of RR intervals (SDNN), square root of the mean of the sum of the squares of differences between adjacent RR intervals (RMSSD), adjacent RR interval differing more than 50ms (NN50) and its percentage denoted by pNN50. All the time domain parameters generally reflect cardiac vagal tone. ${ }^{[26]}$

\section{Cardiac Autonomic Reactivity Tests \\ Heart Rate Response to Standing}

After 10 min of supine rest, recording of lead II ECG was started and the subject was asked to stand quickly with minimal effort, so that artefacts can be minimized. RR tachogram is acquired. From the tachogram, 30:15 ratio is computed, which is the ratio between the longest RR interval at or around $30^{\text {th }}$ beat and the shortest around at or RR interval $15^{\text {th }}$ beat. The 30:15 ratio indicates the parasympathetic reactivity of the heart. ${ }^{[27]}$

\section{Heart Rate Response to Deep Breathing}

The subjects were trained to breath at the rate of six cycles per minute. The procedure involved deep breathing in the sitting posture at the rate of six breaths per minute, with inspiratory and expiratory cycles for 5 sec each, with recording of continuous lead II ECG. Then E:I ratio was calculated, which is the ratio between the maximum RR interval during expiration and the minimum RR interval during inspiration for each breath cycle. This depicts the parasympathetic reactivity of the heart. ${ }^{[27]}$

\section{BP Response to Sustained Handgrip}

This was measured using a handgrip dynamometer (Inco, Ambala). The subject was asked to grip the handgrip dynamometer with maximal force using the dominant hand and the maximal voluntary contraction (MVC) was determined. Then the subject was instructed to maintain $30 \%$ of MVC for three minutes and the BP was measured in the resting arm. The maximum DBP attained during the maneuver was noted. Following the maneuver, blood pressure was recorded 2 min after the release of the grip. The difference between the maximum DBP measured just before release of handgrip and baseline DBP was calculated. Increase in DBP $>15 \mathrm{mmHg}$ above the resting value $(\triangle \mathrm{DBP} \mathrm{isg})$ is taken as a measure of sympathetic reactivity. ${ }^{[28]}$

\section{Recording of Baroreflex Sensitivity (BRS)}

BRS was measured noninvasively, using the equipment Finometer PRO, (Finapres Medical Systems BV, Amsterdam, Netherlands) by using the technique of continuous beat-to-beat $\mathrm{BP}$ variability. Pulse tracing was acquired by finger plethysmography based on the principle of volume clamp technique of Penaz and the Physiocal criteria of Wesseling. ${ }^{[2]}$ Baroreceptor sensitivity was computed as change in inter-beat interval for a unit change in blood pressure. From the continuous recording of reconstructed brachial pressure and inter-beat interval, the baroreflex sensitivity is computed using cross-correlation technique.

The subjects were asked to lie down and the brachial cuff of Finapres was tied around the mid-arm about two $\mathrm{cm}$ above the cubital fossa and the appropriate size finger cuff was tied around the middle phalanx of the middle finger depending on the finger width. For the height correction, two sensors were placed, one at the heart level and another at the finger level, then the cables of the cuffs were connected to the finometer and the recording was done after $10 \mathrm{~min}$ of supine rest. The reconstructed brachial pressure and pulse tracing was acquired by a PC-based data acquisition system Beatscope Easy, (Finapres Medical Systems BV) and BRS was derived.

\section{Measurement of Perceived Stress}

The perceived stress level of the study participants was assessed using the Cohen's Perceived Stress Scale (PSS) - questionnaire. ${ }^{[30]}$ The questionnaire assesses the level to which the participants evaluate various circumstances in their lives as being stressful over the past one month. It is a ten item self-reported scale, four of which are reverse-scored, measured on a 5 -point scale from 0 to 4 . PSS scores were obtained by reversing responses (e.g., $0=4,1=3,2=2,3=1$ and $4=0$ ) to the four positively stated items (items 4, 5, 7 and 8) and then summing across all scale items. Total score ranges from 0 to 40 . The validity and reliability of this questionnaire has been established in various studies. ${ }^{[31]}$

\section{Biochemical Parameters}

Five $\mathrm{ml}$ of venous blood was collected and serum was obtained for uric acid and nitric oxide assay. For nitric oxide, Diagnostic kit from Oxford Biomedical Research was used and nitric oxide was assessed by the method "Non-Enzymatic Colorimetric Assay". Diagnostic kit from Robonik was used to assess uric acid by the method "Automated Photometric assay".

\section{Yoga Intervention}

The subjects of both the study and control groups were not on any medication. Baseline recording of all the study parameters were done in both the groups. Pranayama group received Chandranadi pranayama, which is an exclusive, slow left nostril breathing practice, trained by a qualified yoga instructor at Advanced Centre for Yoga Therapy, Education and Research (ACYTER), JIPMER, Puducherry.

The Yogic intervention protocol followed in this study includes, practice of 27 rounds of chandranadi pranayama followed by 10 -minute relaxation in shavasana in each session. For chandranadi pranayama, practice the subject was made to sit straight and taught to perform nasika mudra. They were asked to close the right nostril by thumb and inhale slowly and deeply through the left nostril, while counting 1-5 in mind and then slowly exhales from the same nostril counting 1-5, this constitute one round of CNP. Few supervised sessions were given initially, once trained in the pranayama, they were asked to practice it at home twice a day, early morning and early evening (preferably in empty stomach or 2 hours after meal). They were asked to maintain an attendance sheet during their practice. Those who completed 12 weeks of pranayama practice with minimum $80 \%$ of attendance were called for post intervention recording of the outcome parameters. The control subjects were neither trained, nor allowed to practice left nostril breathing during the study period. After 12 weeks, the study parameters were recorded in both the groups.

\section{Statistical Analysis of Data}

Statistical analysis was done using SPSS version 21. The data was presented as mean and standard deviation for normally distributed values. Inter group difference of means between control and study group were compared using unpaired t test. Intra group difference of means at baseline and after 12 weeks in control group and study group were compared using paired $t$-test. The difference was considered statistically significant if $p$ value was less than $0.05(p<0.05)$.

\section{RESULTS}

In our RCT, both the groups were of comparable age group, mean age for controls were $32.2 \pm 7.26$ and for study group $32 \pm 6.98(p=0.939)$. Both groups had comparable height $(p=0.841)$, weight $(\mathrm{p}=0.231)$, 
BMI $(p=0.260)$ and WHR $(p=0.402)$. The basal heart rate $(p=0.295)$, resting $\operatorname{SBP}(p=0.722)$ and $\operatorname{DBP}(p=0.268)$ were not significantly different between the groups (Table 1). Therefore, both the groups were comparable in terms of anthropometric and baseline cardiovascular parameters.

In our study (Table 2), compares the cardiovascular parameters, autonomic function tests, biochemical parameters and perceived stress score between 0 and 12 weeks in pranayama group. The basal heart rate $(p=0.004)$, resting systolic and diastolic blood pressure $(p<0.0001)$, mean arterial pressure $(p<0.0001)$, rate pressure product $(p<0.001)$ and PSS $(p=0.001)$ was significantly reduced following 12 weeks of pranayama practice compared to the baseline values, while baroreceptor reflex sensitivity $(p=0.0001)$ was found to be increased following the intervention.

Among the time domain indices of short-term heart rate variability, NN50 $(p=0.007)$ and pNN50 ( $p=0.001)$ have increased significantly. In the frequency domain indices, a significant reduction in the LF/HF ratio ( $p=0.026$ ) was observed following pranayama practice. The cardiac autonomic reactivity parameters, $30: 15$ ratio $(p=0.002)$ and E: I ratio $(p=0.004)$ were significantly increased in the study group. Serum nitric oxide was increased following pranayama practice, whereas uric acid did not show statistical significance.

Table 3 compares cardiovascular parameters, autonomic functions, biochemical parameters and perceived stress score between 0 and 12 weeks in control group subjects. On comparison of all the parameters at baseline and after 12 weeks no statistically significant difference was observed in any of the outcome parameters.

\section{DISCUSSION}

In this randomized controlled trial, we have assessed cardiac autonomic functions, PSS and serum NO, uric acid levels in young adult prehypertensive male subjects. In our study, both groups were comparable in terms of age, height, weight, BMI, resting HR and BP. We have observed a significant reduction in the HR, SBP, DBP and MAP in subjects of the pranayama group. HR is determined by background vagal activity and reduction in the HR denotes enhanced parasympathetic drive to the heart. ${ }^{[32]}$ DBP depends on peripheral vascular resistance (PVR), which in-turn is determined by the vessel diameter, which is under the control of the sympathetic nervous system. ${ }^{[33]}$ As reported earlier, during CNP practice, parasympathetic activity overrides sympathetic nervous system activity, leading to withdrawal of sympathetic tone to the skeletal

Table 1: Comparison of anthropometric and baseline cardiovascular parameters between control group and pranayama group.

$\begin{array}{cccc}\text { Parameter } & \text { Control group } & \text { Pranayama group } & \text { P value } \\ \text { Age (years) } & 32.2 \pm 7.26 & 32 \pm 6.98 & 0.939 \\ \text { Height }(\mathrm{m}) & 1.69 \pm 0.08 & 1.69 \pm 0.06 & 0.841 \\ \text { Weight }(\mathrm{kg}) & 73.6 \pm 11.29 & 69.01 \pm 9.12 & 0.231 \\ \text { BMI }\left(\mathrm{kg} / \mathrm{m}^{2}\right) & 25.55 \pm 2.54 & 24.28 \pm 3.45 & 0.260 \\ \text { WHR } & 0.94 \pm 0.03 & 0.92 \pm 0.07 & 0.402 \\ \text { Basal HR (bpm) } & 75 \pm 14.12 & 79.6 \pm 8.93 & 0.295 \\ \text { SBP }(\mathrm{mm} \mathrm{Hg}) & 127.2 \pm 5.65 & 128 \pm 6.5 & 0.722 \\ \text { DBP }(\mathrm{mm} \mathrm{Hg}) & 79.67 \pm 5.74 & 82.07 \pm 5.9 & 0.268\end{array}$

Values expressed as mean $\pm \mathrm{SD}$; Analysis done by student's unpaired $t$-test. $P<$ 0.05 is considered as statistically significant.

BMI: Body mass index; WHR: Waist-hip ratio; HR: Heart rate; SBP: Systolic blood Pressure; DBP: Diastolic blood pressure
Table 2: Comparison of Cardiovascular Parameters, Autonomic Function Test, Biochemical Parameters and Perceived Stress Score between 0 and 12 Weeks in Pranayama Group.

\begin{tabular}{|c|c|c|c|}
\hline \multirow[t]{2}{*}{ Parameter } & \multicolumn{2}{|c|}{ Pranayama group } & \multirow[t]{2}{*}{$P$ value } \\
\hline & Pre (0 weeks) & Post ( 12 weeks) & \\
\hline \multicolumn{4}{|c|}{ Cardiovascular and PSS parameters } \\
\hline $\begin{array}{c}\text { Basal HR } \\
\text { (bpm) }\end{array}$ & $79.6 \pm 8.93$ & $77.67 \pm 8.11$ & 0.004 \\
\hline $\mathrm{SBP}(\mathrm{mmHg})$ & $128 \pm 6.5$ & $124.87 \pm 6.22$ & $<0.0001$ \\
\hline $\mathrm{DBP}(\mathrm{mmHg})$ & $82.07 \pm 5.9$ & $77.87 \pm 5.89$ & 0.0001 \\
\hline $\begin{array}{c}\mathrm{MAP} \\
(\mathrm{mmHg})\end{array}$ & $97.38 \pm 5.16$ & $93.44 \pm 4.89$ & $<0.0001$ \\
\hline $\mathrm{RPP}$ & $101.18 \pm 1.66$ & $96.98 \pm 2.93$ & $<0.001$ \\
\hline $\begin{array}{c}\text { BRS (ms/ } \\
\mathrm{mmHg} \text { ) }\end{array}$ & $10.91 \pm 6.53$ & $12.32 \pm 6.61$ & 0.0001 \\
\hline PSS & $18.53 \pm 4.85$ & $15.33 \pm 4.32$ & 0.001 \\
\hline \multicolumn{4}{|c|}{ HRV- Time Domain Indices } \\
\hline Mean RR & $780.97 \pm 119.45$ & $829.54 \pm 113.98$ & 0.136 \\
\hline SDNN (ms) & $40.35 \pm 19.04$ & $44.79 \pm 12.11$ & 0.330 \\
\hline RMSSD (ms) & $20.76 \pm 9.49$ & $28.23 \pm 11.81$ & 0.440 \\
\hline NN50 & $8.53 \pm 8.3$ & $21.67 \pm 18.8$ & 0.007 \\
\hline pNN50 (\%) & $2.24 \pm 2.45$ & $5.59 \pm 3.8$ & 0.001 \\
\hline \multicolumn{4}{|c|}{ HRV- Frequency Domain Indices } \\
\hline $\mathrm{TP}\left(\mathrm{ms}^{2}\right)$ & $1223.33 \pm 197.22$ & $2116.2 \pm 156.4$ & $<0.001$ \\
\hline LF nu & $63.11 \pm 12.54$ & $59.16 \pm 9.24$ & 0.108 \\
\hline HF nu & $36.89 \pm 12.54$ & $40.84 \pm 9.24$ & 0.108 \\
\hline $\mathrm{LF} / \mathrm{HF}$ & $1.97 \pm 0.86$ & $1.58 \pm 0.62$ & 0.026 \\
\hline \multicolumn{4}{|c|}{ Cardiac autonomic reactivity tests } \\
\hline $30: 15$ ratio & $1.45 \pm 0.19$ & $1.55 \pm 0.21$ & 0.002 \\
\hline E : I ratio & $1.35 \pm 0.17$ & $1.45 \pm 0.15$ & 0.004 \\
\hline$\Delta \mathrm{DBD}_{\mathrm{IHG}}$ & $19.33 \pm 6.55$ & $17.33 \pm 3.99$ & 0.222 \\
\hline \multicolumn{4}{|c|}{ Biochemical Parameters } \\
\hline Nitric Oxide & $377.73 \pm 9.72$ & $392.01 \pm 10.8$ & $<0.001$ \\
\hline Uric Acid & $7.16 \pm 3.12$ & $6.68 \pm 2.5$ & 0.182 \\
\hline
\end{tabular}

Values expressed as mean $\pm \mathrm{SD}$; Analysis done by student's paired $t$-test.

$P<0.05$ is considered as statistically significant.

SBP: Systolic Blood Pressure, DBP: Diastolic Blood Pressure, PP: Pulse Pressure, MAP: Mean Arterial Pressure, RPP: Rate Pressure Product, BRS: Baroreceptor reflex sensitivity, Mean RR: Mean RR interval, SDNN: Standard Deviation of Normal to Normal interval, RMSSD: Square root of the mean squared differences of successive normal to normal intervals, NN50: the number of interval differences of successive $\mathrm{NN}$ intervals greater than $50 \mathrm{~ms}$, pNN50: the proportion derived by dividing NN50 by the total number of NN I tervals, TP: Total Power, LF nu: Low Frequency component expressed as normalized unit, HF nu: High Frequency component expressed as normalized unit, LF-HF ratio: ratio of low-frequency power to high-frequency power of heart rate variability, 30:15 ratio: Ratio between maximum RR interval at 30 beat and minimum RR interval at $15^{\text {th }}$ beat, E:I ratio: ratio of longest RR interval during expiration to the shortest $R R$ interval during inspiration averaged over 6 cycles of respiration, $\triangle$ DBP: difference between the maximum DBP measured just before release of handgrip and baseline DBP parameters and stress score. 
Table 3: Comparison of Cardiovascular Parameters, Autonomic Functions, Biochemical Parameters and Perceived Stress Score between 0 and 12 Weeks In Control Group.

\begin{tabular}{|c|c|c|c|}
\hline \multirow[t]{2}{*}{ Parameter } & \multicolumn{2}{|c|}{ Control } & \multirow[t]{2}{*}{$P$ value } \\
\hline & Pre (0 weeks) & Post (12 weeks) & \\
\hline \multicolumn{4}{|c|}{ Cardiovascular and PSS parameters } \\
\hline Basal HR (bpm) & $75 \pm 14.12$ & $73.93 \pm 12.57$ & 0.808 \\
\hline SBP (bpm) & $127.2 \pm 5.65$ & $126.2 \pm 5.28$ & 0.165 \\
\hline $\mathrm{DBP}(\mathrm{mmHg})$ & $79.67 \pm 5.74$ & $80.07 \pm 4.53$ & 0.722 \\
\hline MAP (mmHg) & $95.67 \pm 6.16$ & $94.57 \pm 4.54$ & 0.389 \\
\hline RPP & $94.27 \pm 19.24$ & $93.5 \pm 17.09$ & 0.912 \\
\hline $\begin{array}{l}\text { Resting BRS (ms/ } \\
\text { mmHg) }\end{array}$ & $12.97 \pm 5.57$ & $12.51 \pm 5.06$ & 0.755 \\
\hline PSS & $15.93 \pm 5.42$ & $16.4 \pm 4.37$ & 0.7753 \\
\hline \multicolumn{4}{|c|}{ HRV- Time Domain Indices } \\
\hline Mean RR & $881.64 \pm 161.15$ & $869.81 \pm 107.84$ & 0.766 \\
\hline SDNN (ms) & $45.35 \pm 9.96$ & $48.98 \pm 13.5$ & 0.489 \\
\hline RMSSD (ms) & $26.19 \pm 6.42$ & $25.79 \pm 4.24$ & 0.829 \\
\hline NN50 & $21.33 \pm 16.36$ & $21.2 \pm 13.77$ & 0.981 \\
\hline pNN50 (\%) & $6.14 \pm 4.4$ & $5.63 \pm 2.93$ & 0.697 \\
\hline \multicolumn{4}{|c|}{ HRV- Frequency Domain Indices } \\
\hline $\mathrm{TP}$ & $1895.5 \pm 1005.8$ & $2260 \pm 1479.13$ & 0.515 \\
\hline LF nu & $56.83 \pm 15.12$ & $62.62 \pm 6.22$ & 0.141 \\
\hline HF nu & $43.17 \pm 15.12$ & $37.38 \pm 6.23$ & 0.141 \\
\hline $\mathrm{LF} / \mathrm{HF}$ & $1.63 \pm 1.06$ & $1.77 \pm 0.58$ & 0.660 \\
\hline \multicolumn{4}{|c|}{ Cardiac Autonomic Reactivity Tests } \\
\hline $30: 15$ ratio & $1.55 \pm 0.21$ & $1.64 \pm 0.22$ & 0.204 \\
\hline $\mathrm{E}:$ I ratio & $1.45 \pm 0.18$ & $1.35 \pm 0.11$ & 0.147 \\
\hline$\Delta \mathrm{DBD}_{\mathrm{IHG}}$ & $19.6 \pm 7.4$ & $20.33 \pm 4.03$ & 0.783 \\
\hline \multicolumn{4}{|c|}{ Biochemical Parameters } \\
\hline Nitric Oxide & $480.05 \pm 185.06$ & $456.76 \pm 183.7$ & 0.097 \\
\hline Uric Acid & $7.58 \pm 1.24$ & $7.37 \pm 3.83$ & 0.903 \\
\hline
\end{tabular}

Values expressed as mean $\pm \mathrm{SD}$; Analysis done by student's paired $t$-test. $P<$ 0.05 is considered as statistically significant.

muscle blood vessels and decreases the PVR, hence there is reduction in DBP ${ }^{[34]}$ Further, increase in parasympathetic tone also decreases the myocardial chronotropy and dromotropy, with less pronounced effects on inotropy and lusitropy, which is mediated via acetylcholine, released from the parasympathetic nerve ending and its action on $\mathrm{M}_{2}$ muscarinic receptors in the myocardium, resulting in the reduction of $\mathrm{HR}, \mathrm{SBP}$ and MAP in pranayama practicing pre-hypertensive subjects. ${ }^{[35]}$

Rate pressure product is an indirect measure of myocardial oxygen uptake and workload, ${ }^{[36]}$ there was a significant fall in RPP in our study. In the present study, resting BRS was found to be significantly increased following CNP practice. Our finding is in line with Masson et al. ${ }^{[37]}$ BRS, specify the vigor with which the baroreflex arc operates in maintaining the cardiovascular homeostasis, ${ }^{[38]}$ which is computed as the change in HR (millisecond) for a unit change in blood pressure ( $\mathrm{mmHg}$ ).
Decrease in BRS is a predictor of CV risk in hypertensive patients. ${ }^{[39]}$ Slow breathing practice causes reduction in BP and enhancement in baroreflex sensitivity in hypertensive patients. ${ }^{[40]}$ Thus, increased BRS in pranayama group indicates decreased CV risk in them.

In short-term HRV analysis, FDI assessment in the pranayama group revealed a significant reduction in LF-HF ratio, which is a qualitative index of sympathovagal balance. In addition, TP was markedly raised in pranayama practitioners. Cardiac autonomic reactivity tests revealed an improvement in 30:15 and E:I ratio and non-significant decrease in diastolic pressure following hand-grip test. These findings suggest that pre-hypertensive subjects encounter autonomic imbalance in the form of higher adrenergic and reduced vagal drive. Following twelve weeks of CNP practice, we had witnessed a significant improvement in parasympathetic reactivity and relative decrease in sympathetic reactivity and better sympathovagal balance. Our findings are in agreement with Pal GK (2004), Jerath ( 2006 ) and Pramanik (2009), ${ }^{[18,41,42]}$ which revealed that slow deep breathing elevates parasympathetic tone but can affect sympathetic tone differentially depending on the type of slow breathing pattern practiced. In this trial, control group showed no change in the outcome parameters at 0 and 12 weeks.

Perceived stress is found to be reduced in pranayama practitioners. ${ }^{[43]}$ During pranayama practice, the practitioners focus their attention to the act of breathing. The act of concentrating attention in breath de-stress the individuals and this stress-free state of mind evokes parasympathetic dominance and hence enter into a more relaxed phase. ${ }^{[4]}$ Therefore, PSS is found to be reduced in the pranayama group, our findings were consistent with Bhimani and Sharma, et al. ${ }^{[45,46]}$

Furthermore, we have also assessed nitric oxide (NO) and uric acid level in this study, as substantial research evidence suggests the role of endothelial dysfunction with decreased $\mathrm{NO}$ availability in the pathogenesis of hypertension. ${ }^{[47,48]}$ In our study, we observed a significant increase in nitric oxide level in CNP practitioner, indicating increased vascular endothelial functions. Further, $\mathrm{NO}$ is a potent vasodilator that may help to normalize BP. Kuwabara M, et al. has implicated hyperuricemia in new onset hypertension. ${ }^{[49]}$ Hence, we assessed uric acid levels in our subjects, though we noted a fall in serum uric acid, significance was not observed in the intervention arm, may be 12 -week duration of exclusive left nostril breathing practice is not sufficient to cause a change in uric acid levels in our study subjects. In the control group, no significant changes were observed in the various outcome parameters recorded in this study at baseline and after 12 weeks in prehypertensive subjects.

\section{Limitations of the Study}

It is a pilot study, replicating the study in a larger sample can accurately state the effect of 12 weeks CNP practice on biomarkers (NO, Uric acid), autonomic tone and reactivity accurately.

\section{CONCLUSION}

Prehypertension is associated with derangement in autonomic nervous system modulation, in the form of increased sympathetic activity and a decreased vagal activity. An exclusive slow pranayama practice through left nostril (chandranadi pranayama) for 12 weeks was found to restore the sympathovagal balance and reduced the perceived stress in them.

\section{ACKNOWLEDGEMENT}

We would like to sincerely thank the participants of the study and the yoga instructors of ACYTER for supporting this research. 
Aswathy, et al.: Chandranadi Pranayama practice on Cardiac Autonomic Functions and Perceived Stress Score

\section{Sources of funding}

This is an Intramural funded project from Jawaharlal Institute of Postgraduate Medical Education and Research, Pondicherry, India.

\section{CONFLICT OF INTEREST}

Authors declare that they have no conflict of interest.

\section{ABBREVIATIONS}

SBP: Systolic Blood Pressure; DBP: Diastolic Blood Pressure; PP: Pulse Pressure; MAP: Mean Arterial Pressure; RPP: Rate Pressure Product; BRS: Baroreceptor Reflex Sensitivity; Mean RR: Mean RR interval; SDNN: Standard Deviation of Normal to Normal interval; RMSSD: Square root of the mean squared differences of successive normal to normal intervals; NN50: The number of interval differences of successive NN intervals greater than $50 \mathrm{~ms}$; pNN50: The proportion derived by dividing NN50 by the total number of NN intervals; TP: Total Power; LF nu: Low Frequency component expressed as normalized unit; HF nu: High Frequency component expressed as normalized unit.

\section{REFERENCES}

1. Mohan S, Campbell N, Chockalingam A. Time to effectively address hypertension in India. Indian J Med Res. 2013;137(4):627.

2. Gupta R, Gaur K, Ram CVS. Emerging trends in hypertension epidemiology in India. J Hum Hypertens. 2019;33(8):575-87.

3. Chia YC. Prehypertension: What is the current status?. Malays Fam Physician. 2008;3(2):72.

4. Pal GK, Pal P, Nanda N, Lalitha V, Dutta TK, Adithan C. Sympathovagal imbalance in prehypertensive offspring of two parents versus one parent hypertensive. Int J Hypertens. 2011;2011.

5. Parthaje PM, Unnikrishnan B, Thankappan KR, Thapar R, Fatt QK, Oldenburg B. Prevalence and correlates of prehypertension among adults in urban South India. Asia Pac J Public Health. 2016;28(1_suppl):93S-101S.

6. Gupta R, Deedwania PC, Achari V, Bhansali A, Gupta BK, Gupta A, et al. Normotension, prehypertension and hypertension in urban middle-class subjects in India: Prevalence, awareness, treatment and control. Am J Hypertens. 2013;26(1):83-94.

7. Spruill TM. Chronic psychosocial stress and hypertension. Curr Hypertens Rep. 2010;12(1):10-6

8. Rau R. The association between blood pressure and work stress: The importance of measuring isolated systolic hypertension. Work Stress. 2006;20(1):84-97.

9. Ford MT. Perceived unfairness at work, social and personal resources and resting blood pressure. Stress Health. 2014;30(1):12-22.

10. Mucci N, Giorgi G, DePasquale CS, Fiz-Pérez J, Mucci F, Arcangeli G. Anxiety, stress-related factors and blood pressure in young adults. Front Psychol. $2016 ; 7: 1682$

11. Mancia G, Grassi G. The autonomic nervous system and hypertension. Circ Res. 2014;114(11):1804-14

12. Pal GK, Adithan $C$, Amudharaj D, Dutta TK, Pal P, Nandan PG, et al. Assessment of sympathovagal imbalance by spectral analysis of heart rate variability in prehypertensive and hypertensive patients in Indian population. Clin Exp Hypertens. 2011;33(7):478-83.

13. Pal GK, Adithan C, Ananthanarayanan PH, Pal P, Nanda N, Durgadevi T, et al. Sympathovagal imbalance contributes to prehypertension status and cardiovascular risks attributed by insulin resistance, inflammation, dyslipidemia and oxidative stress in first degree relatives of type 2 diabetics. PLoS One. 2013;8(11):e78072

14. Pal GK, Pal P, Nanda N, Amudharaj D, Adithan C. Cardiovascular dysfunctions and sympathovagal imbalance in hypertension and prehypertension: Physiological perspectives. Future Cardiol. 2013;9(1):53-69.

15. Collier SR, Landram MJ. Treatment of prehypertension: lifestyle and/or medication. Vasc Health Risk Manag. 2012;8:613

16. Tyagi $A$, Cohen M. Yoga and heart rate variability: A comprehensive review of the literature. Int J Yoga. 2016;9(2):97.

17. Pal GK. Effects of pranayama on cardiovascular health. Int J Clin Exp Physiol. 2016;3(2):57-8

18. Pal GK, Velkumary S. Effect of short-term practice of breathing exercises on autonomic functions in normal human volunteers. Ind J Med Res. $2004 ; 120(2): 115$
19. Pal GK, Agarwal A, Karthik S, Pal P, Nanda N. Slow yogic breathing through right and left nostril influences sympathovagal balance, heart rate variability and cardiovascular risks in young adults. North Am J Med Sci. 2014;6(3):145.

20. Raghuraj P, Telles S. Immediate effect of specific nostril manipulating yoga breathing practices on autonomic and respiratory variables. Appl Psychophysio Biofeedback. 2008;33(2):65-75.

21. Bhavanani AB, Madanmohan SZ. Immediate effect of Chandra nadi pranayama (left unilateral forced nostril breathing) on cardiovascular parameters in hypertensive patients. Int J Yoga. 2012;5(2):108-11.

22. Ghasemi A, Zahediasl S, Syedmoradi L, Azizi F. Association between serum nitric oxide metabolites and hypertension in a general population. Int J Angiol. 2011;30(4):380-7.

23. Gamboa A, Okamoto LE, Diedrich A, Choi L, Robertson D, Farley G, et al Sympathetic activation and nitric oxide function in early hypertension. Am J Physiol Heart Circ Physiol. 2012;302(7):H1438-43.

24. Hijmering ML, Stroes ES, Olijhoek J, Hutten BA, Blankestijn PJ, Rabelink TJ. Sympathetic activation markedly reduces endothelium-dependent, flowmediated vasodilation. J Am Coll Cardiol. 2002;39(4):683-8.

25. Electrophysiology TF of the ES of $C$ the NAS of P. Heart rate variability: Standards of measurement, physiological interpretation and clinical use. Circulation. 1996;93(5):1043-65

26. Laborde S, Mosley E, Thayer JF. Heart Rate Variability and Cardiac Vagal Tone in Psychophysiological Research - Recommendations for Experiment Planning, Data Analysis and Data Reporting. Front Psychol. 2017;8:213

27. Ewing DJ, Martyn CN, Young RJ, Clarke BF. The value of cardiovascular autonomic function tests: 10 years' experience in diabetes. Diab Care. 1985;8(5):491-8

28. Westerhof BE, Settels JJ, Bos WJW, Westerhof N, Karemaker JM, Wieling W, et al. Bridging cardiovascular physics, physiology and clinical practice: Karel $\mathrm{H}$ Wesseling, pioneer of continuous noninvasive hemodynamic monitoring. Am J Physiol Heart Circ Physiol. 2015;308(3):H153-6.

29. Wesseling KH. Finger arterial pressure measurement with Finapres. Zeitschrift fur Kardiologie. 1996;85:38.

30. Cohen S. Perceived stress in a probability sample of the United States. 1988.

31. Subramanian SK, Sharma VK, Arunachalam V, Rajendran R, Gaur A. Comparison of baroreflex sensitivity and cardiac autonomic function between adolescent athlete and non-athlete boys-A cross sectional study. Front Physiol. 2019; 10:1043.

32. Jose $A D$, Collison $D$. The normal range and determinants of the intrinsic heart rate in man. Cardiovasc Res. 1970;4(2):160-7.

33. Pal GK, Pal P, Nanda N. Comprehensive Textbook of Medical Physiology. Jaypee Medical Ltd. 2016

34. Nivethitha L, Mooventhan A, Manjunath NK. Effects of various Prānāyāma on cardiovascular and autonomic variables. Anc Sci Life. 2016;36(2):72.

35. Bhavanani AB, Shatapathy CC, Sahai A. Modulation of cardiovascular response to exercise by yoga training. Indian J Physiol Pharmacol. 2004;48(4):461-5.

36. Homan TD, Bordes S, Cichowski E. Physiology, Pulse Pressure. In: StatPearls Treasure Island (FL): Stat Pearls Publishing. 2020.

37. Mason H, Vandoni M, Debarbieri G, Codrons E, Ugargol V, Bernardi L. Cardiovascular and respiratory effect of yogic slow breathing in the yoga beginner: what is the best approach?. Evid Based Complement Altern Med. 2013.

38. DiRienzo M, Parati G, Radaelli A, Castiglioni P. Baroreflex contribution to blood pressure and heart rate oscillations: time scales, time-variant characteristics and nonlinearities. Philos Trans A Math Phys Eng Sci. 2009;367(1892):1301-18.

39. DeFerrari GM, Sanzo A, Bertoletti A, Specchia G, Vanoli E, Schwartz PJ. Baroreflex sensitivity predicts long-term cardiovascular mortality afte myocardial infarction even in patients with preserved left ventricular function. $J$ Am Coll Cardiol. 2007:50(24):2285-90.

40. Joseph CN, Porta C, Casucci G, Casiraghi N, Maffeis M, Rossi M, et al. Slow breathing improves arterial baroreflex sensitivity and decreases blood pressure in essential hypertension. Hypertension. 2005;46(4):714-8.

41. Jerath R, Edry JW, Barnes VA, Jerath V. Physiology of long pranayamic breathing: Neural respiratory elements may provide a mechanism that explains how slow deep breathing shifts the autonomic nervous system. Med Hypotheses. 2006;67(3):566-71.

42. Pramanik T, Sharma HO, Mishra S, Mishra A, Prajapati R, Singh S. Immediate effect of slow pace bhastrika pranayama on blood pressure and heart rate. JACM. 2009;15(3):293-5.

43. Ma X, Yue ZQ, Gong ZQ, Zhang H, Duan NY, Shi YT, et al. The effect of diaphragmatic breathing on attention, negative affect and stress in healthy adults. Front Psychol. 2017;8:874.

44. Goyal R, Lata H, Walia L, Narula MK. Effect of pranayama on rate pressure product in mild hypertensives. Int J Appl Basic Med Res. 2014;4(2):67.

45. Bhimani NT, Kulkarni NB, Kowale A, Salvi S. Effect of Pranayama on stress and 
cardiovascular autonomic function. Indian J Physiol Pharmacol. 2011;55(4):3707.

46. Sharma VK, Trakroo M, Subramaniam V, Rajajeyakumar M, Bhavanani AB, Sahai A. Effect of fast and slow pranayama on perceived stress and cardiovascular parameters in young health-care students. Int J Yoga. 2013;6(2):104.

47. Sartori C, Lepori M, Scherrer U. Interaction between nitric oxide and the cholinergic and sympathetic nervous system in cardiovascular control in humans. Pharmacol Therapeutics. 2005;106(2):209-20

48. Hermann M, Flammer A, Lüscher TF. Nitric oxide in hypertension. J Clin Hypertens. 2006;8:17-29.

49. Kuwabara M, Hisatome I, Niwa K, Hara S, Roncal-Jimenez CA, Bjornstad $P$ et al. Uric acid is a strong risk marker for developing hypertension from prehypertension: A 5-year Japanese cohort study. Hypertension. 2018;71(1):7886.

Cite this article: Aswathy V, Velkumary S, Pal GK, Gowsi K. Impact of 12 Weeks Practice of Chandranadi Pranayama on Cardiac Autonomic Functions, Perceived Stress Score and Biochemical Parameters in Pre-hypertensive Young Adult Males- A Pilot Study. Int J Clin Exp Physiol. 2020;7(3):112-8. 